Article

\title{
Cohen Macaulayness and Arithmetical Rank of Generalized Theta Graphs
}

\author{
Seyyede Masoome Seyyedi and Farhad Rahmati *
}

Faculty of Mathematics and Computer Science, Amirkabir University of Technology, 424 Hafez Ave., P.O. Box 15875-4413, Tehran 1591634311, Iran; mseyyedi@aut.ac.ir

* Correspondence: frahmati@aut.ac.ir; Tel.: +98-21-64542534

Academic Editor: Hvedri Inassaridze

Received: 5 March 2016; Accepted: 6 June 2016; Published: 29 June 2016

\begin{abstract}
In this paper, we study some algebraic invariants of the edge ideal of generalized theta graphs, such as arithmetical rank, big height and height. We give an upper bound for the difference between the arithmetical rank and big height. Moreover, all Cohen-Macaulay (and unmixed) graphs of this type will be characterized.
\end{abstract}

Keywords: arithmetical rank; Cohen-Macaulay; height

\section{Introduction}

For an ideal $I$ of a commutative ring $R$ with identity , the arithmetical rank (ara I) of the ideal $I$ is defined as the minimum number $s$ of elements $a_{1}, \ldots, a_{s}$ of $R$ such that $\sqrt{I}=\sqrt{\left(a_{1}, \ldots, a_{s}\right)}$. For a squarefree monomial ideal $I$, it is known that $p d_{R}(R / I) \leq \operatorname{ara}(I)$ and $\operatorname{bight}(I) \leq p d_{R}(R / I)$ (see, for example, [1,2]). Thus,

$$
h t(I) \leq \operatorname{bight}(I) \leq p d_{R}(R / I) \leq \operatorname{ara}(I) \leq \mu(I)
$$

where $\mu(I)$ is the minimum number of generators of $I . R / I$ is Cohen-Macaulay if and only if $h t(I)=p d_{R}(R / I)$. An ideal $I$ is called a set-theoretic complete intersection whenever $h t(I)=\operatorname{ara}(I)$. If $I$ is not unmixed, then $I$ is not a set-theoretic complete intersection. However, it is possible to have $\operatorname{bight}(I)=p d_{R}(R / I)=\operatorname{ara}(I)$. The question then arises, "For which ideal does the previous equality hold?"

Given a polynomial ring $R=K\left[x_{1}, \ldots, x_{n}\right]$ over a field $K$ and a simple graph $G$ with the vertex set $V_{G}=\left\{x_{1}, \ldots, x_{n}\right\}$ and the edge set $E_{G}$, the edge ideal of $G$, denoted by $I(G)$, is the ideal of $R$ generated by $x_{i} x_{j}$ such that $\left\{x_{i}, x_{j}\right\} \in E_{G}$. The graph $G$ is called Cohen-Macaulay over the field $K$ if the ring $R / I(G)$ is Cohen-Macaulay.

It is still an open problem to find an explicit formula for the arithmetical rank of the edge ideal of a graph. For the edge ideal of a forest, it is shown that $\operatorname{bight}(I(G))=\operatorname{ara} I(G)=p d(R / I(G))$ by Barile [3] and Kimura and Terai [4]. In [5], Barile et al. proved that $\operatorname{ara}(G)=p d(R / I(G))$ when $G$ is a cyclic or bicyclic graph. In [6], Mohammadi and Kiani investigated the graphs consisting of some cycles and lines that have a common vertex. It is shown that the projective dimension equals the arithmetical rank for all such graphs. A graph $G$ is called an $n$-cyclic graph with a common edge if $G$ is a graph consisting of $n$ cycles $C_{3 r_{1}+1}, \ldots, C_{3 r_{k_{1}}+1}, C_{3 t_{1}+2}, \ldots, C_{3 t_{k_{2}}+2}, C_{3 s_{1}}, \ldots, C_{3 s_{k_{3}}}$ connected through a common edge, where $k_{1}+k_{2}+k_{3}=n$. Zhu, Shi and Gu proved that $p d(R / I(G))=\operatorname{bight} I(G)=\operatorname{ara}(I(G))$ for some special $n$-cyclic graphs with a common edge [7]. For the class of generalized theta graphs, $G=\theta_{n_{1}, \ldots, n_{k}}$, the authors in [8] showed that $p d(R / I(G))=\operatorname{bight} I(G)$ except in the following two cases: 
1. $n_{i} \equiv 0(\bmod 3)$ for any $1 \leq i \leq k$;

2. there exists exactly one $n_{j}$ such that $n_{j} \equiv 1(\bmod 3)$, and for any $1 \leq i \neq j \leq k$, we have $n_{i} \equiv 2$ $(\bmod 3)$.

For these cases, they show that $p d(R / I(G))=\operatorname{bight} I(G)+1$.

Since bight $I(G) \leq \operatorname{ara}(I(G))$, it can be interesting to compare these invariants for the generalized theta graphs. In the sequel, we compute the height of the edge ideal of generalized theta graphs based on the number of vertices being even or odd in any path. Moreover, we show that $G=\theta_{n_{1}, \ldots, n_{k}}$ is Cohen-Macaulay (and unmixed) if and only if $G=\theta_{2,3,4}$.

\section{Arithmetical Rank of the Edge Ideal of a Generalized Theta Graph}

Let $k \geq 3$ be a positive integer and $n_{1}, \ldots, n_{k}$ be a sequence of positive integers. Let $\theta_{n_{1}, \ldots, n_{k}}$ be the graph constructed by $k$ paths with $n_{1}, \ldots, n_{k}$ vertices with only the endpoints in common. Since the graphs are assumed to be simple, at most one of $n_{1}, \ldots, n_{k}$ can be equal to two. Throughout this paper, we assume that $x$ and $y$ are the common vertices. We define the projective dimension of $G$ to be the projective dimension of the $R$-module $R / I(G)$ and we will write $p d(G)=p d(R / I(G))$. The edge ideal of a cycle of length $n$ with the vertex set $\left\{x_{1}, \ldots, x_{n}\right\}$ is $I\left(C_{n}\right)=\left(x_{1} x_{2}, x_{2} x_{3}, \ldots, x_{n-1} x_{n}, x_{n} x_{1}\right)$. The edge ideal of a line (path) with the vertex set $\left\{x_{1}, \ldots, x_{n}\right\}$ is $I\left(L_{n}\right)=\left(x_{1} x_{2}, x_{2} x_{3}, \ldots, x_{n-1} x_{n}\right)$. In the following, we consider the labeling below:

$$
\begin{gathered}
I\left(L_{3 s_{i}}\right)=\left(x_{1, i} x_{2, i}, x_{2, i} x_{3, i}, \ldots, x_{3 s_{i}-1, i} x_{3 s_{i}, i}\right) ; \text { for } i=1, \ldots, k_{3} \\
I\left(L_{3 r_{j}+1}\right)=\left(y_{1, j} y_{2, j}, y_{2, j} y_{3, j}, \ldots, y_{3 r_{j}, j} y_{3 r_{j}+1, j}\right) ; \text { for } j=1, \ldots, k_{1} \\
I\left(L_{3 t_{l}+2}\right)=\left(z_{1, l} z_{2, l}, z_{2, l} z_{3, l}, \ldots, z_{3 t_{l}+1, l} z_{3 t_{l}+2, l}\right) ; \text { for } l=1, \ldots, k_{2}
\end{gathered}
$$

Suppose that $\min \left\{n_{1}, \ldots, n_{k}\right\}=n_{t}$. One can consider the graph $\theta_{n_{1}, \ldots, n_{k}}$ as a $(k-1)$-cyclic graph with common path $L_{n_{t}}$ consisting of $k-1$ cycles of lengths $n_{i}+n_{t}-2$ for any $1 \leq i \neq t \leq k$. This generalizes the concept of $n$-cyclic graphs with a common edge.

For instance, let $G=\theta_{n_{1}, \ldots, n_{k_{1}+k_{3}}}$ be the graph consisting of lines $L_{3 r_{1}+1}, \ldots, L_{3 r_{k_{1}}+1}, L_{3 s_{1}}, \ldots, L_{3 s_{k_{3}}}$ such that $k_{1}, k_{3}>0$. Without loss of generality, suppose that $\min \left\{n_{1}, \ldots, n_{k_{1}+k_{3}}\right\}=3 s_{1}$. One can consider $G$ as a $\left(k_{1}+k_{3}-1\right)$-cyclic graph with common path $L_{3 s_{1}}$ that the cycles are of lengths $3 s_{1}+3 r_{j}+1-2=3\left(s_{1}+r_{j}-1\right)+2$ or $3 s_{1}+3 s_{i}-2=3\left(s_{1}+s_{i}-1\right)+1$ for $1 \leq j \leq k_{1}$ and $2 \leq i \leq k_{3}$. Consider the following labeling for $I\left(C_{3\left(s_{1}+r_{j}-1\right)+2}\right)$ :

$$
\begin{array}{r}
I\left(C_{3\left(s_{1}+r_{j}-1\right)+2}\right)=\left(x_{1,1} x_{2,1}, \ldots, x_{3 s_{1}-1,1} x_{3 s_{1}, 1}, x_{3 s_{1}, 1} y_{3 s_{1}+1, j}, y_{3 s_{1}+1, j} y_{3 s_{1}+2, j}, \ldots,\right. \\
\left.y_{3\left(s_{1}+r_{j}-1\right)+1, j} y_{3\left(s_{1}+r_{j}-1\right)+2, j}, y_{3\left(s_{1}+r_{j}-1\right)+2, j} x_{1,1}\right)
\end{array}
$$

In this section, we obtain an upper bound for the arithmetical rank of the edge ideal of generalized theta graphs. Using the big height of the edge ideal of these graphs computed in [8], we estimate an upper bound for $\operatorname{araI}\left(\theta_{n_{1}, \ldots, n_{k}}\right)-\operatorname{bight} I\left(\theta_{n_{1}, \ldots, n_{k}}\right)$. For this purpose, we consider seven cases that are treated separately in the following theorems.

Theorem 1. Let $G=\theta_{n_{1}, \ldots, n_{k_{3}}}$ be the graph consisting of lines $L_{3 s_{1}}, \ldots, L_{3 s_{k_{3}}}$, i.e., $n_{i}=3 s_{i}$ for $1 \leq i \leq k_{3}$. Then,

$$
0 \leq \operatorname{araI}(G)-\operatorname{bight} I(G) \leq k_{3}-1
$$

Proof. By definition, one can consider $G$ as a $\left(k_{3}-1\right)$-cyclic graph with common path of length $\min \left\{n_{1}, \ldots, n_{k_{3}}\right\}$. Without loss of generality, we may assume $\min \left\{n_{1}, \ldots, n_{k_{3}}\right\}=3 s_{1}$. 
Since $3 s_{1}+3 s_{i}-2=3\left(s_{1}+s_{i}-1\right)+1$ for any $i \neq 1$, by ([5], Proposition 2.3), we can construct $Q_{2}, \ldots, Q_{k_{3}}$ as follows: for any $2 \leq i \leq k_{3}$, set $Q_{i}=\left(q_{0}, q_{1, i}, q_{2}, \ldots, q_{2\left(s_{1}+s_{i}-1\right)+1, i}\right)$ where

$$
\begin{aligned}
& q_{0}=x_{1,1} x_{2,1} \\
& q_{1, i}=x_{1,1} x_{3\left(s_{1}+s_{i}-1\right)+1, i}+x_{2,1} x_{3,1} \\
& q_{2}=x_{4,1} x_{5,1} \\
& q_{3}=x_{3,1} x_{4,1}+x_{5,1} x_{6,1} \\
& \vdots \\
& q_{2\left(s_{1}-1\right)}=x_{3 s_{1}-2,1} x_{3 s_{1}-1,1} \\
& q_{2 s_{1}-1}=x_{3 s_{1}-3,1} x_{3 s_{1}-2,1}+x_{3 s_{1}-1,1} x_{3 s_{1}, 1} \\
& q_{2 s_{1}, i}=x_{3 s_{1}+1, i} x_{3 s_{1}+3, i} \\
& \vdots \\
& q_{2\left(s_{1}+s_{i}-1\right), i}=x_{3\left(s_{1}+s_{i}-1\right), i} x_{3\left(s_{1}+s_{i}-1\right)+1, i}
\end{aligned}
$$

Observe that the sequences $Q_{2}, \ldots, Q_{k_{3}}$ have $2 s_{1}-1$ common elements, namely $q_{0}, q_{2}, \ldots, q_{2 s_{1}-1}$. On the other hand, by ([9] p. 249), we have $\sqrt{Q_{i}}=I\left(C_{3\left(s_{1}+s_{i}-1\right)+1}\right)$. Therefore, we deduce

$$
\operatorname{araI}(G) \leq\left(2 s_{1}-1\right)+\sum_{i=2}^{k_{3}}\left(\left(2 s_{1}+2 s_{i}-2-2 s_{1}+1\right)+1\right)=2 \sum_{i=1}^{k_{3}} s_{i}-1
$$

Similar to the proof of Theorem 2.11 of [8], we obtain that $\operatorname{bightI}(G)=2 \sum_{i=1}^{k_{3}} s_{i}-k_{3}$, it follows that

$$
0 \leq \operatorname{araI}(G)-\operatorname{bight} I(G) \leq k_{3}-1
$$

as desired.

Theorem 2. Let $G=\theta_{n_{1}, \ldots, n_{k_{1}}}$ be the graph consisting of lines $L_{3 r_{1}+1}, \ldots, L_{3 r_{k_{1}}+1}$, i.e., $n_{i}=3 r_{i}+1$ for $1 \leq i \leq k_{1}$. Then,

$$
p d(G)=\operatorname{bight} I(G)=\operatorname{araI}(G)=2 \sum_{i=1}^{k_{1}} r_{i}
$$

Proof. For $1 \leq i \leq k_{1}$, a similar argument as in ([3], p. 4701), Put $Q_{i}$ is generated up to radical by

$$
\begin{aligned}
& y_{2, i} y_{3, i} \\
& y_{1, i} y_{2, i}+y_{3, i} y_{4, i} \\
& \vdots \\
& y_{3 j-1, i} y_{3 j, i} \\
& y_{3 j-2, i} y_{3 j-1, i}+y_{3 j, i} y_{3 j+1, i} \\
& \vdots \\
& y_{3 r_{i}-2, i} y_{3 r_{i}-1, i}+y_{3 r_{i}, i} y_{3 r_{i}+1, i}
\end{aligned}
$$

we have $I(G)=\sqrt{Q_{1}+\ldots+Q_{k_{1}}}$ by ([9], p. 249). Then, $\operatorname{araI}(G) \leq 2 \sum_{i=1}^{k_{1}} r_{i}$. Similar to the proof of Theorem 2.6 of [8], we obtain that $2 \sum_{i=1}^{k_{1}} r_{i}$, it follows that 


$$
\operatorname{pd}(G)=\operatorname{bight} I(G)=\operatorname{araI}(G)=2 \sum_{i=1}^{k_{1}} r_{i}
$$

Theorem 3. Let $G=\theta_{n_{1}, \ldots, n_{k_{2}}}$ be the graph consisting of lines $L_{3 t_{1}+2}, \ldots, L_{3 t_{k_{2}}+2}$, i.e., $n_{i}=3 t_{i}+2$ for $1 \leq i \leq k_{2}$. Then,

$$
0 \leq \operatorname{ara} I(G)-\operatorname{bight} I(G) \leq k_{2}-2
$$

Proof. We can assume, without loss of generality, that $\min \left\{n_{1}, \ldots, n_{k_{2}}\right\}=n_{1}$. By definition, one may consider $G$ as a $\left(k_{2}-1\right)$-cyclic graph with common path $L_{n_{1}}$ that any cycle contains only $3\left(t_{1}+t_{i}\right)+2$ vertices. Applying Proposition 2.4 of [5], we construct $Q_{i}=\left(q_{0}, q_{1}, \ldots, q_{2\left(t_{1}-1\right)+1}, q_{2 t_{1}, i}, q_{2 t_{1}+1, i}, \ldots, q_{2\left(t_{1}+t_{i}\right), i}\right)$ for any $2 \leq i \leq k_{2}$ as follows:

$$
\begin{aligned}
& q_{0}=z_{1,1} z_{2,1} \\
& q_{1}=z_{2,1} z_{3,1}+z_{4,1} z_{5,1} \\
& \vdots \\
& q_{2 l}=z_{3 l, 1} z_{3 l+1,1}+z_{3 l+2,1} z_{3 l+3,1} \\
& q_{2 l+1}=z_{3 l+2,1} z_{3 l+3,1}+z_{3 l+4,1} z_{3 l+5,1} \\
& \vdots \\
& q_{2\left(t_{1}-1\right)+1}=z_{3 t_{1}-1,1} z_{3 t_{1}, 1}+z_{3 t_{1}+1,1} z_{3 t_{1}+2,1} \\
& q_{2 t_{1}, i}=z_{3 t_{1}, 1} z_{3 t_{1}+1,1}+z_{3 t_{1}+2,1} z_{3 t_{1}+3, i} \\
& \vdots \\
& q_{2\left(t_{1}+h\right), i}=z_{3\left(t_{1}+h\right), i} z_{3\left(t_{1}+h\right)+1, i}+z_{3\left(t_{1}+h\right)+2, i} z_{3\left(t_{1}+h\right)+3, i} \\
& q_{2\left(t_{1}+h\right)+1, i}=z_{3\left(t_{1}+h\right)+2, i} z_{3\left(t_{1}+h\right)+3, i}+z_{3\left(t_{1}+h\right)+4, i} z_{3\left(t_{1}+h\right)+5, i} \\
& \vdots \\
& q_{2\left(t_{1}+t_{i}\right), i}=z_{1,1} z_{3\left(t_{1}+t_{i}\right)+2, i}+z_{3\left(t_{1}+t_{i}\right), i} z_{3\left(t_{1}+h\right)+1, i}
\end{aligned}
$$

We have $I(G)=\sum_{i=2}^{k_{2}} I\left(C_{3\left(t_{1}+t_{i}\right)+2}\right)$, and it follows from ([9], p. 249) that $I(G)=\sqrt{\sqrt{\left(Q_{2}\right)}+\ldots+\sqrt{\left(Q_{k_{2}}\right)}}$. It is easily seen that the sequences $Q_{2}, \ldots, Q_{k_{2}}$ have the terms $q_{0}, q_{1}, \ldots, q_{2\left(t_{1}-1\right)+1}$ in common. Hence,

$$
\operatorname{araI}(G) \leq 2 \sum_{i=1}^{k_{3}} t_{i}+k_{2}-1
$$

Similar to the proof of Theorem 2.7 of [8], we obtain that $\operatorname{bight} I(G)=2 \sum_{i=1}^{k_{2}} t_{i}+1$, and it follows that

$$
0 \leq \operatorname{araI}(G)-\operatorname{bight} I(G) \leq 2 \sum_{i=1}^{k_{2}} t_{i}+k_{2}-1-\left(2 \sum_{i=1}^{k_{2}} t_{i}+1\right)=k_{2}-2
$$

as required.

Theorem 4. Let $G=\theta_{n_{1}, \ldots, n_{k_{1}+k_{3}}}$ be the graph consisting of lines $L_{3 r_{1}+1}, \ldots, L_{3 r_{k_{1}}+1}, L_{3 s_{1}}, \ldots, L_{3 s_{k_{3}}}$, i.e., $n_{i}=3 r_{i}+1$ for $1 \leq i \leq k_{1}$ and $n_{i}=3 s_{i}$ for $k_{1}+1 \leq i \leq k_{1}+k_{3}$ such that $k_{1}, k_{3}>0$. Then, 
1. If there exists $1 \leq i \leq k_{1}$ such that $\min \left\{n_{1}, \ldots, n_{k_{1}+k_{3}}\right\}=3 r_{i}+1$, then $0 \leq \operatorname{araI}(G)-\operatorname{bight} I(G) \leq$ $\min \left\{k_{3}, r_{i}+k_{1}+k_{3}-3\right\}$;

2. If there exists $1 \leq j \leq k_{3}$ such that $\min \left\{n_{1}, \ldots, n_{k_{1}+k_{3}}\right\}=3 s_{j}$, then $0 \leq \operatorname{araI}(G)-\operatorname{bight} I(G) \leq$ $\min \left\{k_{3}, s_{j}+k_{3}-2\right\}$.

Proof. We have $\operatorname{araI}\left(L_{3 r_{i}+1}\right)=2 r_{i}$ and $\operatorname{araI}\left(L_{3 s_{j}}\right)=2 s_{j}$ by ([3], p. 4701). It follows that

$$
\operatorname{araI}(G) \leq 2 \sum_{i=1}^{k_{1}} r_{i}+2 \sum_{j=1}^{k_{3}} s_{j}
$$

1. Without loss of generality, assume that $\min \left\{n_{1}, \ldots, n_{k_{1}+k_{3}}\right\}=3 r_{1}+1$. One can consider $G$ as a $\left(k_{1}+k_{3}-1\right)$-cyclic graph with common path $L_{3 r_{1}+1}$ that the cycles are of lengths $3 r_{1}+1+3 r_{i}+$ $1-2=3\left(r_{1}+r_{i}\right)$ or $3 r_{1}+1+3 s_{j}-2=3\left(r_{1}+s_{j}-1\right)+2$ for $2 \leq i \leq k_{1}$ and $1 \leq j \leq k_{3}$. Now, suppose that

$$
\begin{aligned}
& q_{0}=y_{1,1} y_{2,1} \\
& q_{1, i}=y_{1,1} y_{3\left(r_{1}+r_{i}\right), i}+y_{2,1} y_{3,1} \\
& \vdots \\
& q_{2\left(r_{1}-1\right)+1}=y_{3\left(r_{1}-1\right), 1} y_{3\left(r_{1}-1\right)+1,1}+y_{3\left(r_{1}-1\right)+2,1} y_{3\left(r_{1}-1\right)+3,1} \\
& q_{2 r_{1}, i}=y_{3 r_{1}+1,1} y_{3 r_{1}+2, i} \\
& \vdots \\
& q_{2 h, i}=y_{3 h+1, i} y_{3 h+2, i} \\
& q_{2 h+1, i}=y_{3 h, i} y_{3 h+1, i}+y_{3 h+2, i} y_{3 h+3, i} \\
& \vdots \\
& q_{2\left(r_{1}+r_{i}-1\right)+1, i}=y_{3\left(r_{1}+r_{i}-1\right), i} y_{3\left(r_{1}+r_{i}-1\right)+1, i}+y_{3\left(r_{1}+r_{i}-1\right)+2, i} y_{3\left(r_{1}+r_{i}-1\right)+3, i}
\end{aligned}
$$

Which generate up to radical $I\left(C_{3\left(r_{1}+r_{i}\right)}\right)$. Note that the terms $q_{0}, q_{2}, \ldots, q_{2\left(r_{1}-1\right)+1}$ are in common for any sequences generating ideal $I\left(C_{3\left(r_{1}+r_{i}\right)}\right)$ up to radical and $2 \leq i \leq k_{1}$. For any $1 \leq j \leq k_{3}$, we define:

$$
\begin{aligned}
& q_{0}^{\prime}=y_{1,1} y_{2,1} \\
& q_{1}^{\prime}=y_{2,1} y_{3,1}+y_{4,1} y_{5,1} \\
& \vdots \\
& q_{2 l}^{\prime}=y_{3 l, 1} y_{3 l+1,1}+y_{3 l+2,1} y_{3 l+3,1} \\
& q_{2 l+1}^{\prime}=y_{3 l+2,1} y_{3 l+3,1}+y_{3 l+4,1} y_{3 l+5,1} \\
& \vdots \\
& q_{2\left(r_{1}-1\right)}^{\prime}=y_{3 r_{1}-3,1} y_{3 r_{1}-2,1}+y_{3 r_{1}-1,1} y_{3 r_{1}, 1} \\
& q_{2 r_{1}-1, j}=y_{3 r_{1}-1,1} y_{3 r_{1}, 1}+y_{3 r_{1}+1,1} x_{3 r_{1}+2, j} \\
& \vdots \\
& q_{2 h, j}^{\prime}=x_{3 h, j} x_{3 h+1, j}+x_{3 h+2, j} x_{3 h+3, j} \\
& q_{2 h+1, j}^{\prime}=x_{3 h+2, j} x_{3 h+3, j}+x_{3 h+4, j} x_{3 h+5, j} \\
& \vdots \\
& q_{2\left(r_{1}+s_{j}-1\right), i}^{\prime}=y_{1,1} x_{3\left(r_{1}+s_{j}-1\right)+2, j}+x_{3\left(r_{1}+s_{j}-1\right), j} x_{3\left(r_{1}+s_{j}-1\right)+1, j}
\end{aligned}
$$


and we can obtain that $I\left(C_{3\left(r_{1}+s_{j}-1\right)+2}\right)=\sqrt{\left(q_{0}^{\prime}, q_{1}^{\prime}, \ldots, q_{2\left(r_{1}+s_{j}-1\right), j}\right)}$. We can obtain that there are the common terms $q_{0}^{\prime}, q_{1}^{\prime}, \ldots, q_{2\left(r_{1}-1\right)}^{\prime}$ in any of sequences generating ideal $I\left(C_{3\left(r_{1}+s_{j}-1\right)+2}\right)$ up to radical for any $1 \leq j \leq k_{3}$. On the other hand, we have $q_{0}=q_{0}^{\prime}$ and $q_{2 m+1}=q_{2 m}^{\prime}$, for any $1 \leq m \leq r_{1}-1$. Applying these arguments, we obtain

$$
\operatorname{araI}(G) \leq 2 \sum_{i=1}^{k_{1}} r_{i}+2 \sum_{j=1}^{k_{3}} s_{j}+r_{1}+k_{1}-3
$$

Thus, the inequalities Equations (1) and (2), together with ([8], Theorem 2.8), imply that

$$
0 \leq \operatorname{araI}(G)-\operatorname{bight} I(G) \leq \min \left\{k_{3}, r_{1}+k_{1}+k_{3}-3\right\}
$$

2. We may assume, without loss of generality, that $\min \left\{n_{1}, \ldots, n_{k_{1}+k_{3}}\right\}=3 s_{1}$. One can consider $G$ as a $\left(k_{1}+k_{3}-1\right)$-cyclic graph with common path $L_{3 s_{1}}$ that the cycles are of lengths $3 s_{1}+3 s_{i}-2=$ $3\left(s_{1}+s_{i}-1\right)+1$ for any $2 \leq i \leq k_{3}$ or $3 s_{1}+3 r_{j}+1-2=3\left(s_{1}+r_{j}-1\right)+2$ for any $1 \leq j \leq k_{1}$. Applying Proposition 2.3 of [5], we construct the following sequences:

$$
\begin{aligned}
& q_{0}=x_{1,1} x_{2,1} \\
& q_{1, i}=x_{1,1} x_{3\left(s_{1}+s_{i}-1\right)+1, i}+x_{2,1} x_{3,1} \\
& \vdots \\
& q_{2\left(s_{1}-1\right)}=x_{3 s_{1}-2,1} x_{3 s_{1}-1,1} \\
& q_{2 s_{1}-1}=x_{3 s_{1}-3,1} x_{3 s_{1}-2,1}+x_{3 s_{1}-1,1} x_{3 s_{1}, 1} \\
& q_{2 s_{1}, i}=x_{3 s_{1}+1, i} x_{3 s_{1}+2, i} \\
& \vdots \\
& q_{2 h, i}=x_{3 h+1, i} x_{3 h+2, i} \\
& q_{2 h+1, i}=x_{3 h, i} x_{3 h+1, i}+x_{3 h+2, i} x_{3 h+3, i} \\
& \vdots \\
& q_{2\left(s_{1}+s_{i}-1\right), i}=x_{3\left(s_{1}+s_{i}-1\right), i} x_{3\left(s_{1}+s_{i}-1\right)+1, i}
\end{aligned}
$$

We have $I\left(C_{3\left(s_{1}+s_{i}-1\right)+1}\right)=\sqrt{\left(q_{0}, q_{1, i}, \ldots, q_{2\left(s_{1}+s_{i}-1\right), i}\right)}$ for any $2 \leq i \leq k_{3}$. It is easily seen that the above constructed sequences have $2 s_{1}-1$ terms in common. Now, suppose that $I\left(C_{3\left(s_{1}+r_{j}-1\right)+2}\right)=\sqrt{\left(q_{0}^{\prime}, \ldots, q_{2\left(s_{1}+r_{j}-1\right), j}^{\prime}\right)}$ where 


$$
\begin{aligned}
& q_{0}^{\prime}=x_{1,1} x_{2,1} \\
& q_{1}^{\prime}=x_{2,1} x_{3,1}+x_{4,1} x_{5,1} \\
& \vdots \\
& q_{2 l}^{\prime}=x_{3 l, 1} x_{3 l+1,1}+x_{3 l+2,1} x_{3 l+3,1} \\
& q_{2 l+1}^{\prime}=x_{3 l+2,1} x_{3 l+3,1}+x_{3 l+4,1} x_{3 l+5,1} \\
& \vdots \\
& q_{2\left(s_{1}-1\right)}^{\prime}=x_{3 s_{1}-3,1} x_{3 s_{1}-2,1}+x_{3 s_{1}-1,1} x_{3 s_{1}, 1} \\
& q_{2 s_{1}-1, j}^{\prime}=x_{3 s_{1}-1,1} x_{3 s_{1}, 1}+y_{3 s_{1}+1, j} y_{3 s_{1}+2, j} \\
& \vdots \\
& q_{2 h, j}^{\prime}=y_{3 h, j} y_{3 h+1, j}+y_{3 h+2, j} y_{3 h+3, j} \\
& q_{2 h+1, j}^{\prime}=y_{3 h+2, j} y_{3 h+3, j}+y_{3 h+4, j} y_{3 h+5, j} \\
& \vdots \\
& q_{2\left(s_{1}+r_{j}-1\right), j}^{\prime}=x_{1,1} y_{3\left(s_{1}+r_{j}-1\right)+2, j}+y_{3\left(s_{1}+r_{j}-1\right), j} y_{3\left(s_{1}+r_{j}-1\right)+1, j}
\end{aligned}
$$

for all $1 \leq j \leq k_{1}$. One can check that the above constructed sequences have $2 s_{1}-1$ terms in common. On the other hand, we have $q_{0}=q_{0}^{\prime}$ and $q_{2 m+1}=q_{2 m}^{\prime}$ for any $1 \leq m \leq s_{1}-1$. Using the preceding arguments and the fact that $I(G)=\sum_{i=2}^{k_{3}} I\left(C_{3\left(s_{1}+s_{i}-1\right)+1}\right)+\sum_{j=1}^{k_{1}} I\left(C_{3\left(s_{1}+r_{j}-1\right)+2}\right)$, we get

$$
\operatorname{ara} I(G) \leq 2 \sum_{i=1}^{k_{3}} s_{i}+2 \sum_{j=1}^{k_{1}} r_{j}+s_{1}-2
$$

Thus, the inequalities Equations (1) and (3), together with ([8], Theorem 2.8), yield the inequality

$$
0 \leq \operatorname{araI}(G)-\operatorname{bight} I(G) \leq \min \left\{k_{3}, s_{1}+k_{3}-2\right\}
$$

Theorem 5. Let $G=\theta_{n_{1}, \ldots, n_{k_{2}+k_{3}}}$ be the graph consisting of lines $L_{3 s_{1}}, \ldots, L_{3 s_{k_{3}}}, L_{3 t_{1}+2}, \ldots, L_{3 t_{k_{2}}}+2$, i.e., $n_{i}=3 s_{i}$ for $1 \leq i \leq k_{3}$ and $n_{i}=3 t_{i}+2$ for $k_{3}+1 \leq i \leq k_{3}+k_{2}$ such that $k_{2}, k_{3}>0$. Then,

1. If there exists $1 \leq i \leq k_{3}$ such that $\min \left\{n_{1}, \ldots, n_{k_{2}+k_{3}}\right\}=3 s_{i}$, then $0 \leq \operatorname{araI}(G)-\operatorname{bight} I(G) \leq$ $k_{2}+k_{3}-2$;

2. If there exists $1 \leq j \leq k_{2}$ such that $\min \left\{n_{1}, \ldots, n_{k_{2}+k_{3}}\right\}=3 t_{j}+2$, then $0 \leq \operatorname{araI}(G)-\operatorname{bightI}(G) \leq$ $\min \left\{k_{2}+k_{3}+t_{j}-2, k_{2}+k_{3}-1\right\}$.

\section{Proof.}

1. Without loss of generality, one may assume $\min \left\{n_{1}, \ldots, n_{k_{2}+k_{3}}\right\}=3 s_{1}$. We can consider $G$ as a $\left(k_{2}+k_{3}-1\right)$-cyclic graph with common path $L_{3 s_{1}}$ of which the cycles are of lengths $3 s_{1}+3 s_{i}-2=$ $3\left(s_{1}+s_{i}-1\right)+1$ for any $2 \leq i \leq k_{3}$ or $3 s_{1}+3 t_{j}+2-2=3\left(s_{1}+t_{j}\right)$ for any $1 \leq j \leq k_{2}$. Applying Proposition 2.2 of [5], we have $I\left(C_{3\left(s_{1}+t_{j}\right)}\right)=\sqrt{\left(q_{0}^{\prime}, q_{1, j}^{\prime}, \ldots, q_{2\left(s_{1}+t_{j}\right)-1, j}^{\prime}\right)}$, where 


$$
\begin{aligned}
& q_{0}^{\prime}=x_{1,1} x_{2,1} \\
& q_{1, j}^{\prime}=x_{1,1} z_{3\left(s_{1}+t_{j}\right), j}+x_{2,1} x_{3,1} \\
& \vdots \\
& q_{2\left(s_{1}-1\right)+1}^{\prime}=x_{3\left(s_{1}-1\right), 1} x_{3\left(s_{1}-1\right)+1,1}+x_{3\left(s_{1}-1\right)+2,1} x_{3\left(s_{1}-1\right)+3,1} \\
& q_{2 s_{1}, j}^{\prime}=z_{3 s_{1}+1, j} z_{3 s_{1}+2, j} \\
& \vdots \\
& q_{2 h, j}^{\prime}=z_{3 h+1, j} z_{3 h+2, j} \\
& q_{2 h+1, j}^{\prime}=z_{3 h, j} z_{3 h+1, j}+z_{3 h+2, j} z_{3 h+3, j} \\
& \vdots \\
& q_{2\left(s_{1}+t_{j}-1\right)+1, j}^{\prime}=z_{3\left(s_{1}+t_{j}-1\right), j} z_{3\left(s_{1}+t_{j}-1\right)+1, j}+z_{3\left(s_{1}+t_{j}-1\right)+2, j} z_{3\left(s_{1}+t_{j}-1\right)+3, j}
\end{aligned}
$$

for any $1 \leq j \leq k_{2}$. Note that, for the above constructed sequences, the elements $q_{0}^{\prime}, q_{2}^{\prime}, \ldots, q_{2 s_{1}-1}^{\prime}$ are in common. With the same argument as in the proof of Theorem 4 , we have $I\left(C_{3\left(s_{1}+r_{i}-1\right)+1}\right)=$ $\sqrt{\left(q_{0}, \ldots, q_{2\left(s_{1}+s_{i}-1\right), i}\right)}$ for any $2 \leq i \leq k_{3}$. On the other hand, we have $q_{0}=q_{0}^{\prime}$ and $q_{m}=q_{m}^{\prime}$ for any $2 \leq m \leq 2 s_{1}-1$. In addition, $I(G)=\sum_{i=2}^{k_{3}} I\left(C_{3\left(s_{1}+s_{i}-1\right)+1}\right)+\sum_{j=1}^{k_{2}} I\left(C_{3\left(s_{1}+t_{j}\right)}\right)$. Thus, it follows that

$$
\operatorname{ara}(G) \leq 2 \sum_{i=1}^{k_{3}} s_{i}+2 \sum_{j=1}^{k_{2}} t_{j}+k_{2}-1
$$

Furthermore, ([3], p. 4701) implies that $\operatorname{araI}\left(L_{3 t_{j}+2}\right)=2 t_{j}+1$ and $\operatorname{araI}\left(L_{3 s_{i}}\right)=2 s_{i}$, and hence

$$
\operatorname{ara}(G) \leq 2 \sum_{i=1}^{k_{3}} s_{i}+2 \sum_{j=1}^{k_{2}} t_{j}+k_{2}
$$

From the Equations (4) and (5), together with ([8], Theorem 2.9), we get

$$
0 \leq \operatorname{araI}(G)-\operatorname{bight} I(G) \leq k_{2}+k_{3}-2
$$

as desired.

2. Without loss of generality, one may assume $\min \left\{n_{1}, \ldots, n_{k_{2}+k_{3}}\right\}=3 t_{1}+2$. One can consider $G$ as a $\left(k_{2}+k_{3}-1\right)$-cyclic graph with common path $L_{3 t_{1}+2}$ which the cycles are of lengths $3\left(t_{1}+t_{i}\right)+2$ for any $2 \leq i \leq k_{2}$ or $3\left(t_{1}+s_{j}\right)$ for any $1 \leq j \leq k_{3}$. Using the proof of Theorem 3 , we get $I\left(C_{3\left(t_{1}+t_{i}\right)+2}\right)=\sqrt{\left(q_{0}, \ldots, q_{2\left(t_{1}+t_{i}\right), i}\right)}$ for any $2 \leq i \leq k_{2}$. Assume that 


$$
\begin{aligned}
& q_{0}^{\prime}=z_{1,1} z_{2,1} \\
& q_{1, j}^{\prime}=z_{1,1} x_{3\left(t_{1}+r_{j}\right), j}+z_{2,1} z_{3,1} \\
& \vdots \\
& q_{2\left(t_{1}-1\right)+1}^{\prime}=z_{3\left(t_{1}-1\right), 1} z_{3\left(t_{1}-1\right)+1,1}+z_{3\left(t_{1}-1\right)+2,1} z_{3\left(t_{1}-1\right)+3,1} \\
& q_{2 t_{1, j}}^{\prime}=z_{3 t_{1}+1,1} x_{3 t_{1}+2, j} \\
& \vdots \\
& q_{2 h, j}^{\prime}=x_{3 h+1, j} x_{3 h+2, j} \\
& q_{2 h+1, j}^{\prime}=x_{3 h, j} x_{3 h+1, j}+x_{3 h+2, j} x_{3 h+3, j} \\
& \vdots \\
& q_{2\left(t_{1}+s_{j}-1\right)+1, j}^{\prime}=x_{3\left(t_{1}+s_{j}-1\right), j} x_{3\left(t_{1}+s_{j}-1\right)+1, j}+x_{3\left(t_{1}+s_{j}-1\right)+2, j} x_{3\left(t_{1}+s_{j}-1\right)+3, j}
\end{aligned}
$$

which generate up to radical $I\left(C_{3\left(t_{1}+s_{j}\right)}\right)$. Observe that in all sequences generating ideal $I\left(C_{3\left(t_{1}+s_{j}\right)}\right)$ up to the radical, the elements $q_{0}^{\prime}, q_{2}^{\prime}, \ldots, q_{2\left(t_{1}-1\right)+1}^{\prime}$ are in common, for any $1 \leq j \leq k_{3}$. We have $q_{0}=q_{0}^{\prime}$ and $q_{2 m}=q_{2 m+1}^{\prime}$ for any $1 \leq m \leq t_{1}-1$. Since $I(G)=$ $\sqrt{\sum_{i=2}^{k_{2}} I\left(C_{3\left(t_{1}+t_{i}\right)+2}\right)+\sum_{j=1}^{k_{3}} I\left(C_{3\left(t_{1}+s_{j}\right)}\right)}$,

$$
\operatorname{araI}(G) \leq 2 \sum_{j=1}^{k_{3}} s_{j}+2 \sum_{i=1}^{k_{2}} t_{i}+t_{1}+k_{2}-1
$$

Thus, the inequalities Equations (4) and (6), together with ([8], Theorem 2.9), yield the asserted inequality.

Theorem 6. Let $G=\theta_{n_{1}, \ldots, n_{k_{1}+k_{2}}}$ be the graph consisting of lines $L_{3 r_{1}+1}, \ldots, L_{3 r_{k_{1}+1}}, L_{3 t_{1}+2}, \ldots, L_{3 t_{k_{2}}+2}$, i.e., $n_{i}=3 r_{i}+1$ for $1 \leq i \leq k_{1}$ and $n_{i}=3 t_{i}+2$ for $k_{1}+1 \leq i \leq k_{1}+k_{2}$ such that $k_{1}, k_{2}>0$. Then,

1. If there exists $1 \leq i \leq k_{1}$ such that $\min \left\{n_{1}, \ldots, n_{k_{1}+k_{2}}\right\}=3 r_{i}+1$, then $0 \leq \operatorname{araI}(G)-\operatorname{bight} I(G) \leq$ $\min \left\{2 k_{2}+k_{1}-2, k_{2}\right\}$

2. If there exists $1 \leq j \leq k_{2}$ such that $\min \left\{n_{1}, \ldots, n_{k_{1}+k_{2}}\right\}=3 t_{j}+2$, then $0 \leq \operatorname{araI}(G)-\operatorname{bight} I(G) \leq$ $\min \left\{t_{j}+k_{2}+k_{1}-1, k_{2}\right\}$.

\section{Proof.}

1. Without loss of generality, suppose that $\min \left\{n_{1}, \ldots, n_{k_{1}+k_{2}}\right\}=3 r_{1}+1$. One can consider $G$ as a $\left(k_{1}+k_{2}-1\right)$-cyclic graph with common path $L_{3 r_{1}+1}$ where the cycles are of lengths $3 r_{1}+1+3 t_{j}+$ $2-2=3\left(r_{1}+t_{j}\right)+1$ for any $1 \leq j \leq k_{2}$ or $3 r_{1}+1+3 r_{i}+1-2=3\left(r_{1}+r_{i}\right)$ for any $2 \leq i \leq k_{1}$. Applying the same argument in the proof of Theorem 5 (1), we get

$$
\operatorname{araI}(G) \leq 2 \sum_{i=1}^{k_{1}} r_{i}+2 \sum_{j=1}^{k_{2}} t_{j}+2 k_{2}+k_{1}-2
$$

Hence, ([8], Theorem 2.12), ([3], p. 4701) and Equation (7) imply that $0 \leq \operatorname{araI}(G)-\operatorname{bight} I(G) \leq$ $\min \left\{2 k_{2}+k_{1}-2, k_{2}\right\}$.

2. We may assume, without loss of generality, that $\min \left\{n_{1}, \ldots, n_{k_{1}+k_{2}}\right\}=3 t_{1}+2$. One can consider $G$ as a $\left(k_{1}+k_{2}-1\right)$-cyclic graph with common path $L_{3 t_{1}+2}$. Therefore, the cycles are of lengths 
$3 t_{1}+2+3 t_{i}+2-2=3\left(t_{1}+t_{i}\right)+2$ for any $2 \leq i \leq k_{2}$ or $3 t_{1}+2+3 r_{j}+1-2=3\left(t_{1}+r_{j}\right)+1$ for any $1 \leq j \leq k_{1}$. The same argument as in the proof of Theorem 4 (2) shows that

$$
\operatorname{araI}(G) \leq 2 \sum_{i=1}^{k_{1}} r_{i}+2 \sum_{i=1}^{k_{2}} t_{i}+t_{1}+k_{2}+k_{1}-1
$$

Using ([8], Theorem 2.12), ([3], p. 4701) and Equation (8), one derives that $0 \leq \operatorname{araI}(G)-$ $\operatorname{bight}(G) \leq \min \left\{t_{1}+k_{2}+k_{1}-1, k_{2}\right\}$.

Theorem 7. Let $G=\theta_{n_{1}, \ldots, n_{k_{1}+k_{2}+k_{3}}}$ consist of lines $L_{3 s_{1}}, \ldots, L_{3 s_{k_{3}}}, L_{3 r_{1}+1}, \ldots, L_{3 r_{k_{1}}+1}, L_{3 t_{1}+2}, \ldots, L_{3 t_{k_{2}}+2}$, i.e., $n_{i}=3 s_{i}$ for $1 \leq i \leq k_{3}, n_{i}=3 r_{i}+1$ for $k_{3}+1 \leq i \leq k_{3}+k_{1}$ and $n_{i}=3 t_{i}+2$ for $k_{1}+k_{3}+1 \leq i \leq$ $k_{1}+k_{3}+k_{2}$ such that $k_{1}, k_{2}, k_{3}>0$. Then,

1. If there exists $1 \leq i \leq k_{3}$ such that $\min \left\{n_{1}, \ldots, n_{k_{1}+k_{2}+k_{3}}\right\}=3 s_{i}$, then $0 \leq \operatorname{araI}(G)-\operatorname{bight}(G) \leq$ $\min \left\{k_{2}+k_{3}+s_{i}-2, k_{2}+k_{3}\right\}$;

2. If there exists $1 \leq j \leq k_{1}$ such that $\min \left\{n_{1}, \ldots, n_{k_{1}+k_{2}+k_{3}}\right\}=3 r_{j}+1$, then $0 \leq \operatorname{araI}(G)-$ $\operatorname{bight} I(G) \leq \min \left\{k_{1}+2 k_{2}+k_{3}+r_{i}-3, k_{2}+k_{3}\right\}$;

3. If there exists $1 \leq l \leq k_{2}$ such that $\min \left\{n_{1}, \ldots, n_{k_{1}+k_{2}+k_{3}}\right\}=3 t_{l}+2$, then $0 \leq \operatorname{araI}(G)-\operatorname{bight}(G) \leq$ $\min \left\{k_{1}+k_{2}+k_{3}+t_{l}-1, k_{2}+k_{3}\right\}$.

\section{Proof.}

1. Without loss of generality, suppose that $\min \left\{n_{1}, \ldots, n_{k_{1}+k_{2}+k_{3}}\right\}=3 s_{1}$. One can consider that $G$ is a $\left(k_{1}+k_{2}+k_{3}-1\right)$-cyclic graph with common path $L_{3 s_{1}}$ where the cycles are of lengths $3 s_{1}+3 s_{i}-2=3\left(s_{1}+s_{i}-1\right)+1$ for any $2 \leq i \leq k_{3}$ or $3 s_{1}+3 r_{j}+1-2=3\left(s_{1}+r_{j}-1\right)+2$ for any $1 \leq j \leq k_{1}$ or $3 s_{1}+3 t_{l}+2-2=3\left(s_{1}+t_{l}\right)$ for any $1 \leq l \leq k_{2}$. Using the same argument as in the proof of Theorems 1, 4 (2) and 5 (1), we get

$$
\begin{aligned}
\operatorname{araI}(G) \leq & \left(2 s_{1}-1\right)+2 \sum_{l=1}^{k_{2}} t_{l}+k_{2} \\
& +\left(2 s_{1}-1\right)+2 \sum_{i=2}^{k_{3}} s_{i}+\left(2 s_{1}-1\right)+2 \sum_{j=1}^{k_{1}} r_{j} \\
& -\left(1+2 s_{1}-2\right)-\left(1+s_{1}-1\right) \\
& =2 \sum_{l=1}^{k_{2}} t_{l}+2 \sum_{j=1}^{k_{1}} r_{j}+2 \sum_{i=1}^{k_{3}} s_{i}+k_{2}+s_{1}-2
\end{aligned}
$$

It follows from Equation (9), ([8], Theorem 2.10) and ([3], p. 4701) that

$$
0 \leq \operatorname{ara} I(G)-\operatorname{bight} I(G) \leq \min \left\{k_{2}+k_{3}+s_{1}-2, k_{2}+k_{3}\right\}
$$

2. Without loss of generality, assume that $\min \left\{n_{1}, \ldots, n_{k_{1}+k_{2}+k_{3}}\right\}=3 r_{1}+1$. One can consider that $G$ is a $\left(k_{1}+k_{2}+k_{3}-1\right)$-cyclic graph with common path $L_{3 r_{1}+1}$ where the cycles are of lengths $3\left(r_{1}+r_{j}\right)$ for any $2 \leq j \leq k_{1}, 3\left(r_{1}+t_{l}\right)+1$ for any $1 \leq l \leq k_{2}$ or $3\left(r_{1}+s_{i}-1\right)+2$ for any $1 \leq i \leq k_{3}$. The same argument as in the proof of Theorems 4 (1) and 6 (1) shows that 


$$
\begin{aligned}
\operatorname{araI}(G) \leq & \left(2 r_{1}-1\right)+2 \sum_{l=1}^{k_{1}} r_{l}+k_{1}-1 \\
& +\left(2 r_{1}-1\right)+2 \sum_{j=2}^{k_{2}} t_{j}+2 k_{2}+\left(2 r_{1}-1\right)+2 \sum_{i=1}^{k_{3}} s_{i} \\
& -\left(2 r_{1}-1\right)-\left(1+r_{1}-1\right) \\
& =2 \sum_{l=1}^{k_{2}} t_{l}+2 \sum_{j=1}^{k_{1}} r_{j}+2 \sum_{i=1}^{k_{3}} s_{i}+2 k_{2}+k_{1}+r_{1}-3
\end{aligned}
$$

Therefore, by Equation (10), ([8], Theoerem 2.10) and ([3], p. 4701), we conclude that

$$
0 \leq \operatorname{araI}(G)-\operatorname{bight}(G) \leq \min \left\{k_{1}+2 k_{2}+k_{3}+r_{1}-3, k_{2}+k_{3}\right\}
$$

3. Without loss of generality, assume that $\min \left\{n_{1}, \ldots, n_{k_{1}+k_{2}+k_{3}}\right\}=3 t_{1}+2$. One can consider that $G$ is a $\left(k_{1}+k_{2}+k_{3}-1\right)$-cyclic graph with common path $L_{3 t_{1}+2}$, where the cycles are of lengths $3\left(t_{1}+s_{i}\right)$ for any $1 \leq i \leq k_{3}, 3\left(t_{1}+r_{j}\right)+1$ for any $1 \leq j \leq k_{1}$ or $3\left(t_{1}+t_{l}\right)+2$ for any $2 \leq l \leq k_{2}$. We can use the same argument as in the proof of Theorems 3,5 (2) and 6 (2) to obtain

$$
\begin{aligned}
\operatorname{araI}(G) \leq & 2 t_{1}+2 \sum_{i=1}^{k_{3}} s_{i} \\
& +2 t_{1}+2 \sum_{j=1}^{k_{1}} r_{j}+k_{1}+2 t_{1}+2 \sum_{l=2}^{k_{2}} t_{l}+\left(k_{2}-1\right) \\
& -2 t_{1}-\left(1+t_{1}-1\right) \\
& =2 \sum_{l=1}^{k_{2}} t_{l}+2 \sum_{j=1}^{k_{1}} r_{j}+2 \sum_{i=1}^{k_{3}} s_{i}+t_{1}+k_{2}+k_{1}-1
\end{aligned}
$$

Applying Equation (11), ([8], Theoerem 2.10) and ([3], p. 4701), we get

$$
0 \leq \operatorname{araI}(G)-\operatorname{bight} I(G) \leq \min \left\{k_{1}+k_{2}+k_{3}+t_{1}-1, k_{2}+k_{3}\right\}
$$

as desired.

\section{Cohen-Macaulayness of Generalized Theta Graph}

In [2], Mohammadi and Kiani investigated some properties of graphs of the form $\theta_{n_{1}, \ldots, n_{k}}$ such as shellability, vertex decomposability and sequential Cohen-Macaulayness. The present section is devoted to study Cohen-Macaulayness and unmixedness of these graphs, especially the height of generalized theta graphs. The most important motivation to study this property comes from the fact that $R / I$ is Cohen-Macaulay if and only if $h t(I)=p d(R / I)$. We check the equality $h t I(G)=p d(R / I(G))$ to verify Cohen-Macaulayness of the graph $G=\theta_{n_{1}, \ldots, n_{k}}$ in some cases. Since the projective dimension of a graph in this class is computed in [8], it only remains to obtain the value of $h t I(G)$. 
Let us fix some notations that will be used throughout this section. By $G_{1} \cup G_{2}$, we mean the graph obtaind by the disjoint union of $G_{1}$ and $G_{2}$. Furthermore, we suppose that the vertices of a line graph $L_{n_{i}}$ are labeled by $x_{1, i}, x_{2, i}, \ldots, x_{n_{i}, i}$ where $x_{1, i}=x$ and $x_{n_{i}, i}=y$. Note that

$$
h t\left(I\left(L_{n}\right)\right)= \begin{cases}k & n=2 k \\ k & n=2 k+1\end{cases}
$$

Lemma 8. Let $G=\theta_{n_{1}, \ldots, n_{k}}$ such that $n_{i}=2 m_{i}$ for any $1 \leq i \leq k$. Then,

$$
h t I(G)=\left(\sum_{i=1}^{k} m_{i}\right)-k+1
$$

Proof. Assume that $A$ is a minimal vertex cover of $G$. One of the following cases may happen: $(x \in A, y \notin A),(x \notin A, y \in A),(x, y \in A)$, or $(x, y \notin A)$.

1. Suppose that $x \in A, y \notin A$. We are going to find the minimum cardinality of minimal vertex cover of $G$ which do not contain $y$, so it suffices to cover the disjoint lines $L_{n_{1}-2}, \ldots, L_{n_{k}-2}$ with the minimum number of vertices such that $N_{G}(y) \subseteq A$. We claim that

$$
h t I\left(L_{n_{1}-2} \cup \ldots \cup L_{n_{k}-2}\right)=\sum_{i=1}^{k}\left(m_{i}-1\right)
$$

Note that $n_{i}-2=2\left(m_{i}-1\right)$. Furthermore, there exists a minimal vertex cover $B_{i}$ for $L_{n_{i}-2}$ with the minimum cardinality $m_{i}-1$ such that $x_{2, i} \notin B_{i}$ and $x_{3, i}, x_{n_{i}-1, i} \in B_{i}$ for any $1 \leq i \leq k$. Hence, in this case, the minimum number of vertices of such $A$ to be equal to

$$
1+\sum_{i=1}^{k}\left(m_{i}-1\right)=\left(\sum_{i=1}^{k} m_{i}\right)-k+1
$$

The number 1 appears in the above equality because $x \in A$.

2. Suppose that $y \in A, x \notin A$. We can apply the same argument as in the previous case.

3. Assume that $x, y \in A$. To obtain a minimal vertex cover of $G$ with minimum cardiality, we may cover the disjoint lines $L_{n_{1}-2}, \ldots, L_{n_{k}-2}$ with the minimum number of vertices such that $N_{G}(x)$ and $N_{G}(y)$ are not contained in $A$. Since $n_{i}-2=2\left(m_{i}-1\right)$ and there exists a minimal vertex cover $B_{i}$ for $L_{n_{i}-2}$ with the minimum cardinality $m_{i}-1$ such that $x_{2, i}, x_{n_{i}-1, i} \notin B_{i}$ for any $1 \leq i \leq k$, we deduce that

$$
h t I\left(L_{n_{1}-2} \cup \ldots \cup L_{n_{k}-2}\right)=\sum_{i=1}^{k}\left(m_{i}-1\right)
$$

It follows that the minimum cardinality of such $A$ to be equal to

$$
2+\sum_{i=1}^{k}\left(m_{i}-1\right)=\left(\sum_{i=1}^{k} m_{i}\right)-k+2
$$

The number 2 appears in the above equality because $x, y \in A$.

4. Assume that $x, y \notin A$. Applying the same argument, we may cover the disjoint lines $L_{n_{1}-2}, \ldots, L_{n_{k}-2}$ with the minimum number of vertices such that $A$ contains $N_{G}(x)$ and $N_{G}(y)$. There exists a minimal vertex cover $B_{i}$ of cardinality $m_{i}$ for the line $L_{n_{i}-2}$ having an even number of vertices such that $x_{2, i}, x_{n_{i}-1, i} \in B_{i}$ for any $1 \leq i \leq k$; therefore, we obtain the minimum number of vertices of such $A$ to be equal to $\sum_{i=1}^{k} m_{i}$ because $x, y \notin A$.

Now, by comparing the above cases, we get 


$$
h t I(G)=\left(\sum_{i=1}^{k} m_{i}\right)-k+1
$$

as desired.

Lemma 9. Let $G=\theta_{n_{1}, \ldots, n_{k}}$ such that $n_{i}=2 m_{i}+1$ for any $1 \leq i \leq k$. Then,

$$
h t I(G)=\left(\sum_{i=1}^{k} m_{i}\right)-k+2
$$

Proof. The techniques used in this proof are similar to the previous lemma. Suppose that $A$ is a minimal vertex cover of $G$. The only possible cases for $x, y$ are $(x \in A, y \notin A),(x \notin A, y \in A)$, $(x, y \in A)$, or $(x, y \notin A)$.

1. Suppose that $x \in A, y \notin A$. We have $N_{G}(y) \subseteq A$. There exists a minimal vertex cover $B_{i}$ for the line $L_{n_{i}-2}$ having an odd number of vertices such that $x_{2, i} \notin B_{i}$ and $x_{n_{i}-1, i} \in B_{i}$ where the minimum cardinality of $B_{i}$ is $m_{i}$ for any $1 \leq i \leq k$. Therefore,

$$
h t I\left(L_{n_{1}-2} \cup \ldots \cup L_{n_{k}-2}\right)=\sum_{i=1}^{k} m_{i}
$$

Hence, we get the minimum number of vertices of such $A$ to be equal to

$$
1+\sum_{i=1}^{k} m_{i}
$$

The number 1 appears in the above equality because $x \in A$.

2. Suppose that $y \in A, x \notin A$. We can apply the same argument as in the previous case.

3. Suppose that $x, y \in A$. There exists a minimal vertex cover $B_{i}$ for $L_{n_{i}-2}$ such that $x_{2, i}, x_{n_{i}-1, i} \notin$ $B_{i}$, and, furthermore, the minimum number vertices of such $B_{i}$ is $m_{i}-1$ for any $1 \leq i \leq k$. Since $n_{i}-2=2\left(m_{i}-1\right)+1$,

$$
h t I\left(L_{n_{1}-2} \cup \ldots \cup L_{n_{k}-2}\right)=\left(\sum_{i=1}^{k} m_{i}\right)-k
$$

It follows that the minimum cardinality of such $A$ is equal to

$$
2+\sum_{i=1}^{k}\left(m_{i}-1\right)=\left(\sum_{i=1}^{k} m_{i}\right)-k+2
$$

The number 2 appears in the above equality because $x, y \in A$.

4. Suppose that $x, y \notin A$. Applying the same argument, we may cover the disjoint lines $L_{n_{1}-2}, \ldots, L_{n_{k}-2}$ with the minimum number of vertices such that $A$ contains $N_{G}(x)$ and $N_{G}(y)$. There exists a minimal vertex cover $B_{i}$ of cardinality $m_{i}$ for the line $L_{n_{i}-2}$ having an odd number of vertices such that $x_{2, i}, x_{n_{i}-1, i} \in B_{i}$ for any $1 \leq i \leq k$; therefore, we obtain the minimum number of vertices of such $A$ to be equal to $\sum_{i=1}^{k} m_{i}$, because $x, y \notin A$.

Since $k \geq 3,2-k<0$. Now, we compare the results obtained from the cases above to get

$$
h t I(G)=\left(\sum_{i=1}^{k} m_{i}\right)-k+2
$$

as required. 
Lemma 10. Let $G=\theta_{n_{1}, \ldots, n_{k}}$ such that $n_{i}=2 m_{i}$ for any $1 \leq i \leq k_{1}$ and $n_{i}=2 l_{i}+1$ for any $k_{1}+1 \leq i \leq k$. Then,

$$
h t I(G)=\left(\sum_{i=1}^{k_{1}} m_{i}\right)+\left(\sum_{i=k_{1}+1}^{k} l_{i}\right)-k+2
$$

Proof. Assume that $A$ is a minimal vertex cover for $G$. Applying the same argument in lemma 8, the only possible cases for the common vertices $x, y$ are $(x \in A, y \notin A),(x \notin A, y \in A),(x, y \in A)$, or $(x, y \notin A)$.

1. Assume that $x \in A, y \notin A$. We have to cover the disjoint lines $L_{n_{1}-2}, \ldots, L_{n_{k}-2}$ with the minimum number of vertices such that $N_{G}(y) \subseteq A$. We have $n_{i}-2=2\left(m_{i}-1\right)$ for $1 \leq i \leq k_{1}$ and $n_{i}-2=2\left(l_{i}-1\right)+1$ for $k_{1}+1 \leq i \leq k$. It is easily seen that there exists a minimal vertex cover $B_{i}$ for $L_{n_{i}-2}$ having an even number of vertices, and the minimal vertex cover $C_{i}$ for $L_{n_{i}-2}$ having an odd number of vertices such that $x_{2, i} \notin B_{i}$ for $1 \leq i \leq k_{1}, x_{2, i} \notin C_{i}$ for $k_{1}+1 \leq i \leq k, x_{n_{i}-1, i} \in B_{i}$ for $1 \leq i \leq k_{1}$ and $x_{n_{i}-1, i} \in C_{i}$ for $k_{1}+1 \leq i \leq k_{1}$. The minimum size for $B_{i}$ and $C_{i}$ is $m_{i}-1$ and $l_{i}$, respectively. Hence, in this case, we need at least $\sum_{i=1}^{k_{1}}\left(m_{i}-1\right)+\sum_{i=k_{1}+1}^{k} l_{i}$ vertices to create a minimal vertex cover for the disjoint lines $L_{n_{1}-2}, \ldots, L_{n_{k}-2}$. Then, the minimum size of such $A$ is

$$
\left(\sum_{i=1}^{k_{1}} m_{i}\right)+\left(\sum_{i=k_{1}+1}^{k} l_{i}\right)-k_{1}+1
$$

2. Assume that $y \in A, x \notin A$. We can apply the same argument as in the previous case.

3. Assume that $x, y \in A$. To obtain the minimum cardinality of such $A$, it suffices to cover the disjoint graphs $L_{n_{1}-2}, \ldots, L_{n_{k}-2}$ with the minimum number of vertices such that $x_{2, i}, x_{n_{i}-1, i} \notin A$ for any $1 \leq i \leq k$. There exists a minimal vertex cover $B_{i}$ for the line having an even number of vertices $L_{n_{i}-2}$ with the minimum number of vertices $m_{i}-1$ such that $x_{2, i}, x_{n_{i}-1, i}$ for $1 \leq i \leq k_{1}$ are not contained in $A$. Moreover, there exists a minimal vertex cover $C_{i}$ for the line having an odd number of vertices $L_{n_{i}-2}$ with the minimum number of vertices $l_{i}-1$ which does not contain $x_{2, i}, x_{n_{i}-1, i}$ for $k_{1}+1 \leq i \leq k$. With this argument, to make a minimal vertex cover with the minimum number vertices for the disjoint lines $L_{n_{1}-2}, \ldots, L_{n_{k}-2}$ we may have

$$
\sum_{i=1}^{k_{1}}\left(m_{i}-1\right)+\sum_{i=k_{1}+1}^{k}\left(l_{i}-1\right)
$$

vertices. Then, the minimum cardinalty of such $A$ is equal to

$$
\begin{aligned}
& \sum_{i=1}^{k_{1}}\left(m_{i}-1\right)+\sum_{i=k_{1}+1}^{k}\left(l_{i}-1\right)+2= \\
& \left(\sum_{i=1}^{k_{1}} m_{i}\right)+\left(\sum_{i=k_{1}+1}^{k} l_{i}\right)-k_{1}-\left(k-k_{1}\right)+2= \\
& \left(\sum_{i=1}^{k_{1}} m_{i}\right)+\left(\sum_{i=k_{1}+1}^{k} l_{i}\right)-k+2
\end{aligned}
$$

4. Assume that $x, y \notin A$. In this case, we may cover the disjoint lines $L_{n_{1}-2}, \ldots, L_{n_{k}-2}$ with the minimum number of vertices such that $A$ contains $N_{G}(x)$ and $N_{G}(y)$. For any line $L_{n_{i}-2}$ $\left(1 \leq i \leq k_{1}\right)$ containing even number of vertices, we can find the minimal vertex cover $B_{i}$ with the minimum number of vertices $m_{i}$ which contains $x_{2, i}, x_{n_{i}-1, i}$ for $1 \leq i \leq k_{1}$. In addition, there exists a minimal vertex cover $C_{i}$ with the minimum number of vertices $l_{i}$ which contains $x_{2, i}, x_{n_{i}-1, i}$ for 
$k_{1}+1 \leq i \leq k$. Therefore, in this case, the minimum number of vertices for covering the disjoint lines $L_{n_{1}-2}, \ldots, L_{n_{k}-2}$ is equal to $\sum_{i=1}^{k_{1}} m_{i}+\sum_{i=k_{1}+1}^{k} l_{i}$. Hence, the minimum cardinality of such $A$ equals

$$
\sum_{i=1}^{k_{1}} m_{i}+\sum_{i=k_{1}+1}^{k} l_{i}
$$

Since $k \geq 3$ and $k_{1} \geq 1,1-\left(k-k_{1}\right) \leq 0$. We compare the results obtained from the above cases to obtain

$$
h t I(G)=\left(\sum_{i=1}^{k_{1}} m_{i}\right)+\left(\sum_{i=k_{1}+1}^{k} l_{i}\right)-k+2
$$

as desired.

To verify Cohen-Macaulayness and unmixedness of the generalized theta graphs, we consider only seven possible cases that are described in the following theorems.

Theorem 11. Let $G$ be the graph $\theta_{n_{1}, \ldots, n_{k_{1}}}$ consisting of lines $L_{3 r_{1}+1}, \ldots, L_{3 r_{k_{1}}+1}$, i.e., $n_{i}=3 r_{i}+1$ for $1 \leq i \leq k_{1}$. Then, $G$ is not unmixed and hence not Cohen-Macaulay.

Proof. Applying Theorem 2.6 of [2], one can conclude that $G$ is not sequentially Cohen-Macaulay. This implies $G$ is neither Cohen-Macaulay by ([10], Lemma 3.6) nor unmixed by ([8], Theorem 2.14).

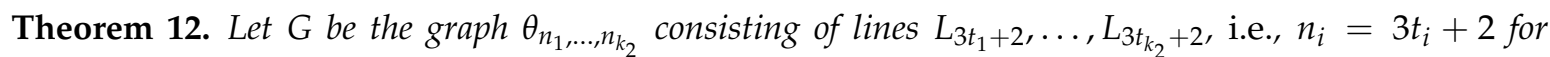
$1 \leq i \leq k_{2}$. Then, $G$ is not unmixed and hence not Cohen-Macaulay.

Proof. It suffices to show that $G$ is not unmixed. We distinguish the three following cases:

1. There exist positive integers $m_{1}, \ldots, m_{k_{2}}$ such that $n_{i}=2 m_{i}$ for any $1 \leq i \leq k_{2}$;

2. There exist nonnegative integers $r_{1}, \ldots, r_{k_{2}}$ such that $n_{i}=2 r_{i}+1$ for any $1 \leq i \leq k_{2}$;

3. There exist positive integers $m_{1}, \ldots, m_{k}$ such that $n_{i}=2 m_{i}$ for any $1 \leq i \leq k$ and nonnegative integer numbers $r_{k+1}, \ldots, r_{k_{2}}$ such that $n_{i}=2 r_{i}+1$ for any $k+1 \leq i \leq k_{2}$.

Using proof of Lemmas 8-10, it is readily seen that there exist two minimal vertex covers of different sizes in any case, and then $G$ is not unmixed.

Theorem 13. Let $G$ be the graph $\theta_{n_{1}, \ldots, n_{k_{1}+k_{3}}}$ consisting of lines $L_{3 r_{1}+1}, \ldots, L_{3 r_{k_{1}}+1}, L_{3 s_{1}}, \ldots, L_{3 s_{k_{3}}}$, i.e., $n_{i}=3 r_{i}+1$ for $1 \leq i \leq k_{1}$ and $n_{i}=3 s_{i}$ for $k_{1}+1 \leq i \leq k_{1}+k_{3}$ such that $k_{1}, k_{3}>0$. Then, G is not unmixed and hence not Cohen-Macaulay.

Proof. We have to verify the following cases:

- The set $\left\{n_{1}, \ldots, n_{k_{1}+k_{3}}\right\}$ does not contain $\{3,4\}$. Applying Theorem 2.6 of [2], $G$ is not sequentially Cohen-Macaulay, and then $G$ is not Cohen-Macaulay by ([10], Lemma 3.6). We therefore get $G$ is not unmixed by ([8], Theorem 2.14).

- $\quad\{3,4\} \subseteq\left\{n_{1}, \ldots, n_{k_{1}+k_{3}}\right\}$. There exist positive integers $m_{i}$ such that $n_{i}=2 m_{i}$ for any $1 \leq i \leq s$ and there exist nonnegative integers $l_{i}$ such that $n_{i}=2 l_{i}+1$ for any $s+1 \leq i \leq k_{1}+k_{3}$. Using cases 3 and 4 of Lemma 10, we obtain two minimal vertex covers $A$ and $B$ of cardinalities $2+\left(\sum_{i=2}^{s} m_{i}\right)+1+\left(\sum_{i=s+2}^{k_{1}+k_{3}} l_{i}\right)-\left(k_{1}+k_{3}\right)+2$ and $2+\left(\sum_{i=2}^{s} m_{i}\right)+1+\sum_{i=s+2}^{k_{1}+k_{3}} l_{i}$, respectively. Since $k_{1}+k_{3} \geq 3,5-\left(k_{1}+k_{3}\right) \neq 3$. Hence, $G$ is not unmixed. Moreover, $G$ is not Cohen-Macaulay.

Theorem 14. Let $G$ be the graph $\theta_{n_{1}, \ldots, n_{k_{2}+k_{3}}}$ consisting of lines $L_{3 s_{1}}, \ldots, L_{3 s_{k_{3}}}, L_{3 t_{1}+2}, \ldots, L_{3 t_{k_{2}}}+2$, i.e., $n_{i}=3 s_{i}$ for $1 \leq i \leq k_{3}$ and $n_{i}=3 t_{i}+2$ for $k_{3}+1 \leq i \leq k_{3}+k_{2}$ such that $k_{2}, k_{3}>0$. Then, $G$ is not unmixed and hence not Cohen-Macaulay. 
Proof. It suffices to replace $\{3,4\}$ by $\{2,3\}$ in the proof of Theorem 13 and apply the same argument.

Theorem 15. Let $G$ be the graph $\theta_{n_{1}, \ldots, n_{k_{3}}}$ consisting of lines $L_{3 s_{1}}, \ldots, L_{3 s_{k_{3}}}$, i.e., $n_{i}=3 s_{i}$ for $1 \leq i \leq k_{3}$. Then, $G$ is not unmixed and hence not Cohen-Macaulay.

Proof. In order to show that $G$ is not unmixed, we use the same argument of Theorem 12.

Theorem 16. Let $G$ be the graph $\theta_{n_{1}, \ldots, n_{k_{1}+k_{2}}}$ consisting of lines $L_{3 r_{1}+1}, \ldots, L_{3 r_{k_{1}}+1}, L_{3 t_{1}+2}, \ldots, L_{3 t_{k_{2}}}+2$, i.e., $n_{i}=3 r_{i}+1$ for $1 \leq i \leq k_{1}$ and $n_{i}=3 t_{i}+2$ for $k_{1}+1 \leq i \leq k_{1}+k_{2}$ such that $k_{1}, k_{2}>0$. Then, $G$ is not unmixed and hence not Cohen-Macaulay.

Proof. From ([2], Lemma 2.6), we obtain that $G$ is not sequentially Cohen-Macaulay and hence $G$ is not Cohen-Macaulay by ([10], Lemma 3.6). Applying Theorem 2.14 of [8], one concludes that $G$ is not unmixed for $k_{1} \geq 2$. To complete the proof, it remains to prove that $G$ is not unmixed for $k_{1}=1$. In this case, the same argument of Theorem 12 holds.

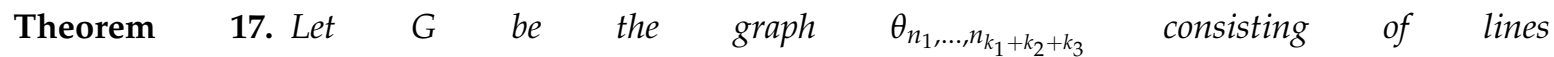
$L_{3 r_{1}+1}, \ldots, L_{3 r_{k_{1}}+1}, L_{3 t_{1}+2}, \ldots, L_{3 t_{k_{2}}+2}, L_{3 s_{1}}, \ldots, L_{3 s_{k_{3}}}$, i.e., $n_{i}=3 r_{i}+1$ for $1 \leq i \leq k_{1}, n_{i}=3 t_{i}+2$ for $k_{1}+1 \leq i \leq k_{1}+k_{2}$ and $n_{i}=3 s_{i}$ for $k_{1}+k_{2}+1 \leq i \leq k_{1}+k_{2}+k_{3}$ such that $k_{1}, k_{2}, k_{3}>0$. Then, $G$ is Cohen-Macaulay (unmixed) if and only if $G=\theta_{2,3,4}$.

Proof.

$\Leftarrow)$ Suppose that $G=\theta_{2,3,4}$. Set $I\left(L_{2}\right)=(x y), I\left(L_{3}\right)=(x e, e y)$ and $I\left(L_{4}\right)=(x z, z t, t y)$. Using CoCoA, $I(G)$ has the minimal primary decomposition as

$$
I(G)=(y, z, e) \cap(x, y, t) \cap(x, y, z) \cap(x, t, e)
$$

Hence, $G$ is unmixed and Cohen-Macaulay by ([8], Theorem 2.14).

$\Rightarrow$ ) We know that $G$ is Cohen-Macaulay (and hence unmixed) if and only if $h t I(G)=p d(G)$. It is not difficult to see that $h t I(G)$ changes according to being even or odd the numbers $r_{i}, t_{j}, s_{m}$ $\left(1 \leq i \leq k_{1}, 1 \leq j \leq k_{2}, 1 \leq m \leq k_{3}\right)$. By the description given above, there are only nine possible cases. By checking all cases, it is seen that the equality $h t I(G)=p d(G)$ holds only for one case. In the following, we examine two cases that seem more important.

- Suppose that there are nonnegative integers $r_{i}, t_{j}$ and $s_{m}$ such that $r_{i}=2 l_{i}+1, t_{j}=2 g_{j}$ and $s_{m}=2 h_{m}+1$ for any $1 \leq i \leq k_{1}, 1 \leq j \leq k_{2}$ and $1 \leq m \leq k_{3}$. By Lemma 10, we get

$$
\begin{aligned}
h t I(G) & =\sum_{i=1}^{k_{1}}\left(3 l_{i}\right)+\sum_{j=1}^{k_{2}}\left(3 g_{j}\right)+\sum_{m=1}^{k_{3}}\left(3 h_{m}\right)+k_{1}+2 \\
& =\frac{3}{2} \sum_{i=1}^{k_{1}} r_{i}+\frac{3}{2} \sum_{j=1}^{k_{2}} t_{j}+\frac{3}{2} \sum_{m=1}^{k_{3}} s_{m}-\frac{k_{1}}{2}-\frac{3}{2} k_{3}+2
\end{aligned}
$$

Similar to the proof of Theorem 2.10 of [8], we obtain that $p d(G)=2 \sum_{i=1}^{k_{1}} r_{i}+2 \sum_{j=1}^{k_{2}} t_{j}+$ $2 \sum_{m=1}^{k_{3}} s_{m}-k_{3}$. Applying Theorem 2.10 of [8], we have 


$$
\begin{aligned}
h t I(G)=p d(G) & \Longleftrightarrow \frac{3}{2} \sum_{i=1}^{k_{1}} r_{i}+\frac{3}{2} \sum_{j=1}^{k_{2}} t_{j}+\frac{3}{2} \sum_{m=1}^{k_{3}} s_{m}-\frac{k_{1}}{2}-\frac{3}{2} k_{3}+2=2 \sum_{i=1}^{k_{1}} r_{i}+2 \sum_{j=1}^{k_{2}} t_{j}+2 \sum_{m=1}^{k_{3}} s_{m}-k_{3} \\
& \Longleftrightarrow \frac{1}{2} \sum_{i=1}^{k_{1}} r_{i}+\frac{1}{2} \sum_{j=1}^{k_{2}} t_{j}+\frac{1}{2} \sum_{m=1}^{k_{3}} s_{m}=2-\frac{k_{1}}{2}-\frac{k_{3}}{2} \\
& \Longleftrightarrow \sum_{i=1}^{k_{1}} r_{i}+\sum_{j=1}^{k_{2}} t_{j}+\sum_{m=1}^{k_{3}} s_{m}=4-k_{1}-k_{3} \\
& \Longleftrightarrow k_{1}=k_{3}=1 \Longleftrightarrow r_{1}+\sum_{j=1}^{k_{2}} t_{j}+s_{1}=2 \\
& \Longleftrightarrow r_{1}=s_{1}=1, k_{2}=1, t_{1}=0 \\
& \Longleftrightarrow G=\theta_{2,3,4}
\end{aligned}
$$

- $\quad$ Suppose that there exist nonnegative integers $l_{i}, g_{j}$ and $h_{m}$ such that $r_{i}=2 l_{i}$ for any $1 \leq i \leq \alpha$ and $r_{i}=2 l_{i}+1$ for any $\alpha+1 \leq i \leq k_{1}, t_{j}=2 g_{j}$ for any $1 \leq j \leq \beta$ and $t_{j}=2 g_{j}+1$ for any $\beta+1 \leq j \leq k_{2}, s_{m}=2 h_{m}$ for any $1 \leq m \leq \gamma$ and $s_{m}=2 h_{m}+1$ for any $\gamma+1 \leq m \leq k_{3}$ which at least one of $\alpha, \beta$ and $\gamma$ is non zero. Note that we choose $\alpha, \beta$ and $\gamma$ such that any of the other cases do not occur. Using Lemma 10, we obtain

$$
\begin{aligned}
h t I(G) & =\sum_{i=1}^{\alpha}\left(3 l_{i}\right)+\sum_{i=\alpha+1}^{k_{1}}\left(3 l_{i}\right)+\sum_{j=1}^{\beta}\left(3 g_{j}\right)+\sum_{j=\beta+1}^{k_{2}}\left(3 g_{j}\right)+\sum_{m=1}^{\gamma}\left(3 h_{m}\right)+\sum_{m=\gamma+1}^{k_{3}}\left(3 h_{m}\right) \\
& +k_{1}+k_{2}-2 \alpha-\beta-\gamma+2=\frac{3}{2} \sum_{i=1}^{k_{1}} r_{i}+\frac{3}{2} \sum_{j=1}^{k_{2}} t_{j}+\frac{3}{2} \sum_{m=1}^{k_{3}} s_{m} \\
& -\frac{k_{1}}{2}-\frac{k_{2}}{2}-\frac{3}{2} k_{3}+2+\frac{\beta}{2}+\frac{\gamma}{2}-\frac{\alpha}{2}
\end{aligned}
$$

Applying Theorem 2.10 of [8], we have

$$
\begin{aligned}
h t I(G)=p d(G) & \Longleftrightarrow \frac{3}{2} \sum_{i=1}^{k_{1}} r_{i}+\frac{3}{2} \sum_{j=1}^{k_{2}} t_{j}+\frac{3}{2} \sum_{m=1}^{k_{3}} s_{m}-\frac{k_{1}}{2}-\frac{k_{2}}{2}-\frac{3}{2} k_{3}+\frac{\beta}{2}+\frac{\gamma}{2}-\frac{\alpha}{2}+2 \\
& =2 \sum_{i=1}^{k_{1}} r_{i}+2 \sum_{j=1}^{k_{2}} t_{j}+2 \sum_{m=1}^{k_{3}} s_{m}-k_{3} \\
& \Longleftrightarrow \frac{1}{2} \sum_{i=1}^{k_{1}} r_{i}+\frac{1}{2} \sum_{j=1}^{k_{2}} t_{j}+\frac{1}{2} \sum_{m=1}^{k_{3}} s_{m}=2+\frac{(\beta+\gamma-\alpha)}{2}-\frac{\left(k_{1}+k_{2}+k_{3}\right)}{2} \\
& \Longleftrightarrow \sum_{i=1}^{k_{1}} r_{i}+\sum_{j=1}^{k_{2}} t_{j}+\sum_{m=1}^{k_{3}} s_{m}=4+\left(\beta-k_{2}\right)+\left(\gamma-k_{3}\right)-\left(\alpha+k_{1}\right)
\end{aligned}
$$

By assumption, we have $\beta-k_{2} \leq 0, \gamma-k_{3} \leq 0$ and $\alpha+k_{1} \geq 1$. Then, it follows that $4+\left(\beta-k_{2}\right)+\left(\gamma-k_{3}\right)-\left(\alpha+k_{1}\right) \leq 3$. Furthermore, we know $\sum_{i=1}^{k_{1}} r_{i}+\sum_{j=1}^{k_{2}} t_{j}+\sum_{m=1}^{k_{3}} s_{m} \geq 2$. Assume $\sum_{i=1}^{k_{1}} r_{i}+\sum_{j=1}^{k_{2}} t_{j}+\sum_{m=1}^{k_{3}} s_{m}=3$. Since $r_{i}>0, t_{j} \geq 0$ and $s_{m}>0$, we conclude $\left(r_{1}=s_{1}=t_{1}=1\right),\left(r_{1}=1, t_{1}=0, s_{1}=2\right)$ or $\left(r_{1}=2, t_{1}=0, s_{1}=1\right)$ which are contradictions by assumption. Suppose that $\sum_{i=1}^{k_{1}} r_{i}+\sum_{j=1}^{k_{2}} t_{j}+\sum_{m=1}^{k_{3}} s_{m}=2$, then we have $r_{1}=1, t_{1}=0$ and $s_{1}=1$. This implies that $\alpha=\gamma=0$ and $\beta=1$, a contradiction. Hence, $G$ is not Cohen-Macaulay (unmixed).

By considering the nine previous theorems, we get the following result: 
Corollary 18. Let $G=\theta_{n_{1}, \ldots, n_{k}}$. Then, the following conditions are equivalent:

(a) $G$ is Cohen-Macaulay;

(b) $G$ is unmixed;

(c) $G=\theta_{2,3,4}$.

\section{Conclusions}

We have shown that algebraic invariants of the ideals associated to combinatorial structers are computable.

Author Contributions: This work is a part of PhD thesis of the first author under supervision of the second.

Conflicts of Interest: The authors declare no conflict of interest.

\section{References}

1. Lyubeznik, G. On the local cohomology modules $H_{A}^{i}(R)$ for ideals $A$ generated by monomials in an R-sequence. Lect. Notes Math. 1984, 1092, 214-220.

2. Mohammadi, F.; Kiani, D. Sequentially Cohen-Macaulay graphs of form $\theta_{n_{1}, \ldots, n_{k}}$. Bull. Iran. Math. Soc. 2010, 36, 109-118.

3. Barile, M. On the arithmetical rank of the edge ideals of forests. Commun. Algebra 2008, 36, 4678-4703.

4. Kimura, K.; Terai, N. Binomial arithmetical rank of edge ideals of forests. Proc. Am. Math. Soc. 2013, 141, 1925-1932.

5. Barile, M.; Kiani, D.; Mohammadi, F.; Yassemi, S. Arithmetical rank of the cyclic and bicyclic graphs. J. Algebra Appl. 2012, 11, 1250039.

6. Mohammadi, F.; Kiani, D. On the arithmetical rank of the edge ideals of some graphs. Algebra Colloq. 2012, 19, 797-806.

7. Zhu, G.; Shi, F.; Gu, Y. Arithmetical rank of the edge ideals of some $n$-cyclic graphs with a common edge. Turk. J. Math. 2015, 39, 112-123.

8. Seyyedi, S.M.; Rahmati, F. Regularity and projective dimension of the edge ideal of generalized theta graph. Turk. J. Math., submitted for publication, 2015.

9. Schmitt, T.; Vogel, W. Note on set-theoretic intersections of subvarieties of projective space. Math. Ann. 1979, 245, 247-253.

10. Francisco, C.A.; van Tuyl, A. Sequentially Cohen-Macaulay edge ideals. Proc. Am. Math. Soc. 2007, 135, 2327-2337.

(C) 2016 by the authors; licensee MDPI, Basel, Switzerland. This article is an open access article distributed under the terms and conditions of the Creative Commons Attribution (CC-BY) license (http://creativecommons.org/licenses/by/4.0/). 